\title{
Does the threshold representation associated with the autoconversion process matter?
}

\author{
H. Guo ${ }^{1}$, Y. Liu ${ }^{1}$, and J. E. Penner ${ }^{2}$ \\ ${ }^{1}$ Atmospheric Science Division, Brookhaven National Laboratory, Upton, NY, USA \\ ${ }^{2}$ Department of Atmospheric, Oceanic and Space Sciences, the University of Michigan, Ann Arbor, MI, USA \\ Received: 24 October 2007 - Published in Atmos. Chem. Phys. Discuss.: 14 November 2007 \\ Revised: 29 January 2008 - Accepted: 2 February 2008 - Published: 4 March 2008
}

\begin{abstract}
Different ad hoc threshold functions associated with the autoconversion process have been arbitrarily used in atmospheric models. However, it is unclear how these ad hoc functions impact model results. Here systematic investigations of the sensitivities of climatically-important properties: CF (cloud fraction), LWP (liquid water path), and AIE (aerosol indirect effect) to threshold functions have been performed using a 3-D cloud-resolving model. It is found that the effect of threshold representations is larger on instantaneous values than on daily averages; and the effect depends on the percentage of clouds in their transitional stages of converting cloud water to rain water. For both the instantaneous values and daily averages, the sensitivity to the specification of critical radius is more significant than the sensitivity to the "smoothness" of the threshold representation (as embodied in the relative dispersion of droplet size distribution) for drizzling clouds. Moreover, the impact of threshold representations on the AIE is stronger than that on CF and LWP.
\end{abstract}

\section{Introduction}

The autoconversion process generally refers to the process whereby droplets grow into embryonic raindrops. It determines the onset of precipitation of warm clouds, influences the precipitation amount, and thereby the global hydrological cycle. Furthermore, the autoconversion process is an important bridge between aerosols, clouds, and precipitation, in that the suppression of precipitation by aerosols could increase cloud spatial and temporal extent (Albrecht, 1989; Pincus and Baker, 1994). Therefore, an appropriate repre-

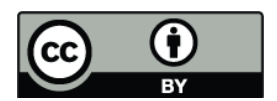

Correspondence to: $\mathrm{H}$. Guo (hguo@bnl.gov) sentation of the autoconversion process is critical for advancing our scientific understanding of cloud micro- and macroproperties, as well as cloud and/or aerosol effects on climate (Boucher et al., 1995; Rotstayn and Liu, 2005).

All the autoconversion parameterizations that have been developed so far can be generically written as

$P=P_{0} T$,

where $P$ is the autoconversion rate $\left(\mathrm{g} \mathrm{cm}^{-3} \mathrm{~s}^{-1}\right), P_{0}$ is the rate function describing the conversion rate after the onset of the autoconversion process, and $T(0 \leq T \leq 1)$ is the threshold function describing the threshold behavior of the autoconversion process. To date, the primary foci of both parameterization development (Kessler, 1969; Manton and Cotton, 1977; Liu and Daum, 2004) and sensitivity investigations (Iacobellis and Somerville, 2006) have been on the rate function $P_{0}$.

The threshold function has received little attention. In most global climate models (GCMs) and/or cloud resolving models (CRMs), the threshold behavior has been represented by an ad hoc function of liquid water content or droplet size. It ranges from an all-or-nothing Kessler-type ( $\mathrm{T}=$ Heaviside function, Kessler, 1969) to a smoother Sundqvist-type ( $\mathrm{T}=$ exponential function, Sundqvist, 1978; Del Genio et al., 1996) and to a constant Berry-type ( $\mathrm{T}=1$, Berry, 1968; Beheng, 1994). Despite their dramatic differences, these functions have been used arbitrarily, and no systematic investigation has been performed to examine whether or not these different representations exert significant effects on model results.

To fill this gap, this study explores how the climatically important properties, i.e. cloud fraction $(\mathrm{CF})$, liquid water path (LWP), and aerosol indirect effect (AIE), respond to different threshold representations by applying a theoretical threshold function to a 3-D cloud-resolving model, ATHAM

Published by Copernicus Publications on behalf of the European Geosciences Union. 
(Active Tracer High-resolution Atmospheric Model) (Herzog et al., 1998, 2003; Guo et al., 2007a).

\section{Threshold representation}

Liu et al. (2006a) derived a theoretical threshold function $\left(T_{\varepsilon}\right)$ that covers all the existing types of threshold representations. Briefly, $T_{\varepsilon}$ is described by

$$
\begin{aligned}
T_{\varepsilon}= & \gamma\left(6 \varepsilon+1,[\Gamma(3 \varepsilon+1)]^{1 /(3 \varepsilon)} x_{c}^{1 /(3 \varepsilon)}\right) \\
& \times \gamma\left(3 \varepsilon+1,[\Gamma(3 \varepsilon+1)]^{1 /(3 \varepsilon)} x_{c}^{1 /(3 \varepsilon)}\right),
\end{aligned}
$$

where $\Gamma(a)=\int_{0}^{\infty} t^{a-1} e^{-t} d t$ is the Gamma function; $\gamma(a, x)=\int_{x}^{\infty} t^{a-1} e^{-t} d t / \int_{0}^{\infty} t^{a-1} e^{-t} d t$ is the normalized incomplete Gamma function; $\varepsilon$ is the relative dispersion (the ratio of the standard deviation to the mean radius of the cloud droplet size distribution); and $x_{c}$ is the ratio of the critical to the mean mass concentration of cloud liquid water (Liu et al., 2006a). Equation (2) indicates that $T_{\varepsilon}$ depends on two dimensionless variables, $\varepsilon$ and $x_{c}$, as compared to ad hoc threshold functions which depend only on $x_{c}$. It should be emphasized that $\varepsilon$ controls the "type" of $T_{\varepsilon}$, changing from the Kessler-type to the Berry-type as $\varepsilon$ increases from 0 to infinity. This dependence of $T_{\varepsilon}$ on $\varepsilon$ allows us to systematically examine the effect of the "smoothness" of the threshold function, which has been unknowingly buried in arbitrary uses of ad hoc threshold functions in previous studies (Liu et al., 2006a).

\section{Model and case descriptions}

ATHAM is a non-hydrostatic, fully compressible atmospheric model. In this study, the 3-D version is adopted. The horizontal domain is $6.4 \times 6.4 \mathrm{~km}^{2}$ with a uniform horizontal spacing of $100 \mathrm{~m}$. The vertical spacing is $30 \mathrm{~m}$ within the boundary layer (below $2 \mathrm{~km}$ ). The time step is $2 \mathrm{~s}$, and model results have been archived every 5 (model) min. The entire simulation period is $30 \mathrm{~h}$, but we mainly examined the model results in the daytime from 06:00 to 18:00 LT (local time) when the AIE is significant. A double-moment cloud microphysical parameterization is employed; cloud droplet number concentration is predicted following Lohmann et al. (1999). Both the shortwave and longwave radiative heating/cooling rates are computed interactively. The model is initialized and driven by the re-analysis data from European Centre for Medium-Range Weather Forecasts (ECMWF).

We investigated two contrasting clean and polluted cases observed during the Second Aerosol Characterization Experiment (ACE-2) over Portugal, the Azores, and the Canary Islands. The clean case (denoted as "CLEAN") occurred on 26 June 1997, when the ACE-2 area was under the control of a cyclone that brought in pristine maritime air. The polluted case (denoted as "POLL") occurred on 9 July 1997, when the ACE-2 area was under the influence of the Azores High that brought in anthropogenic pollution from Europe (Verver et al., 2000). For the "CLEAN" and "POLL" cases, the total aerosol number concentrations were $218 \mathrm{~cm}^{-3}$ and $636 \mathrm{~cm}^{-3}$, respectively (Snider and Brenguier, 2000); and the non sea-salt (nss) sulfate mass concentrations were $0.30 \mu \mathrm{g} \mathrm{m}^{-3}$ and $2.8 \mu \mathrm{g} \mathrm{m}^{-3}$, respectively (Guibert et al., 2003). Note that the detailed model set-up and case descriptions are fully described in Guo et al. (2007a, b).

In addition to the contrasting aerosol and meteorological conditions, significant proportions of these clouds were in their transitional stages where precipitation depended critically on the threshold representation, providing a good opportunity to explore the effects of different threshold representations on model results.

\section{Sensitivity studies}

\subsection{Sensitivity to the relative dispersion $(\varepsilon)$}

To explore all the existing threshold functions, we performed simulations with $\varepsilon=0,0.1,0.2,0.4,0.6,0.8,1.0,10.0$, and 300 in Eq. (2). Note: $\varepsilon=0,0.4$, and 300 approximately represent the Kessler-type, the Sundqvist-type, and the Berry-type threshold functions, respectively. The results are shown in Fig. 1, where the runs for $\varepsilon=0$ are used as the base cases (black), and the results of sensitivity tests for $\varepsilon=0.4$ and 300 are shown as percentage differences relative to the base cases. For the "CLEAN" case, both CF and LWP reach their maxima in the local early morning and their minima in the local afternoon (Fig. 1a and c). But for the "POLL" case, neither CF nor LWP exhibits a significant diurnal cycle due to a strong large-scale subsidence associated with the Azores High (Fig. $1 \mathrm{~b}$ and d). The magnitude of the AIE is the largest near local noon for both the "CLEAN" and "POLL" cases due to the peak insolation (Fig. 1e and f). The "CLEAN" case has a larger AIE than the "POLL" case because the "CLEAN" clouds are deeper and moister (Pawlowska and Brenguier, 2003). Note that CF here is defined as the fraction of cloudy columns, and a cloudy column is a column containing one or more cloudy cells with liquid water mixing ratio $>0.01 \mathrm{~g} / \mathrm{kg}$. The AIE (shortwave plus longwave) at the top-of-the-atmosphere is estimated by swapping the aerosol and meteorological conditions between the "CLEAN" and "POLL" cases and then calculating the radiative flux difference between the clean and polluted aerosol conditions under the same meteorological background (Guo et al., 2007b).

The relative differences in the instantaneous CF, LWP, and AIE between the base cases $(\varepsilon=0)$ and the sensitivity tests $(\varepsilon=300)$ vary significantly, and can reach up to $\sim 20 \%$, $\sim 40 \%$, and $\sim 60 \%$, respectively (Fig. 1). The maximum of the AIE difference can reach $100 \%$ although this only occurs 

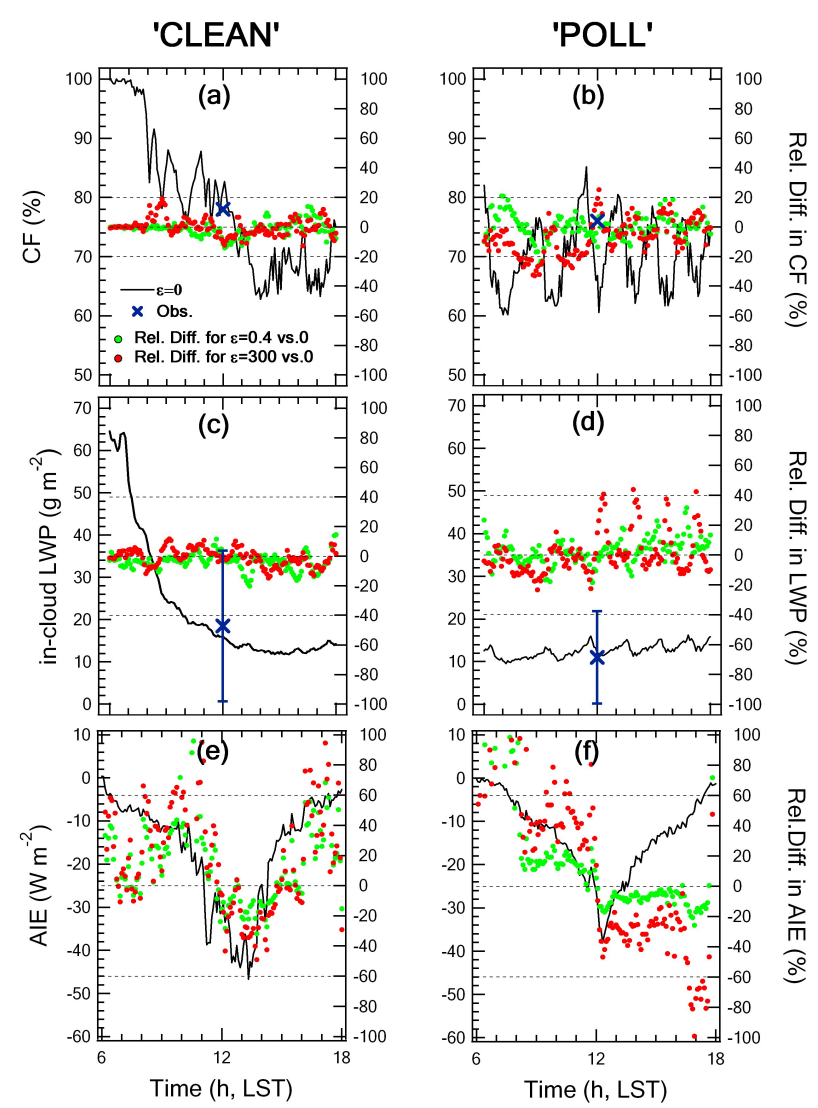

Fig. 1. Time series of cloud fraction $(\mathrm{CF}, \mathbf{a}, \mathbf{b})$, in-cloud liquid water path (LWP, c, d), and aerosol indirect effect (AIE, e, f) at the top-of-the-atmosphere for the base cases ( $\varepsilon=0$, black); and the corresponding relative differences (Rel. Diff.) between the base cases and the sensitivity tests $(\varepsilon=0.4$, green; $\varepsilon=300$, red). The left and right panels are for the "CLEAN" (a, c, e) and "POLL" (b, d, f) cases, respectively. The observed values and their uncertainties are indicated with " $x$ " and vertical bars, respectively, if available.

when the AIE is small. As expected, the difference between $\varepsilon=0$ and 300 is generally larger than that between $\varepsilon=0$ and 0.4. The effect of $\varepsilon$ is stronger for the "POLL" case than that for the "CLEAN" case.

To further explore the underlying physics, Fig. 2 shows the relationship of the relative differences in CF, LWP, and AIE (for $\varepsilon=0$ and 300) as a function of $x_{c}$. It is clear that larger differences in these three quantities are generally associated with larger values of $x_{c}$, suggesting that an exact representation of the threshold behavior becomes more important as the autoconversion becomes less efficient. This is expected because all threshold functions gradually approach 1 as $x_{c}$ decreases to 0 . The value of $x_{c}$ is an increasing function of cloud droplet number concentration $\left(N_{d}\right)$ but a decreasing function of liquid water content (LWC) $\left(x_{c} \sim N_{d}^{3 / 2} \mathrm{LWC}^{-2}\right.$, Eq. (12) of Liu et al., 2005). In the "POLL" case, $x_{c}$ is larger because the $N_{d}$ is larger and the LWC is smaller. Further-

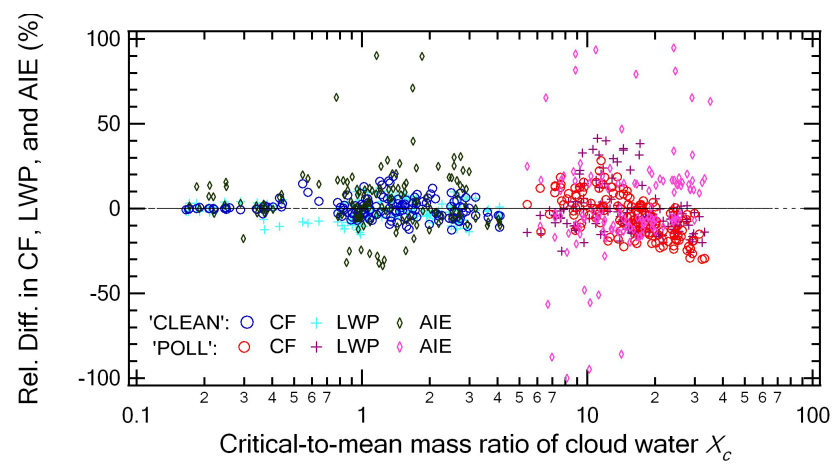

Fig. 2. The relative differences (Rel. Diff.) between the base cases $(\varepsilon=0)$ and the sensitivity tests $(\varepsilon=300)$ vs. the critical-to-mean mass ratio of cloud water $\left(x_{c}\right)$ averaged over all cloudy cells at the same model time for the "CLEAN" and "POLL" cases. The circle, plus, and diamond denote the instantaneous cloud fraction $(\mathrm{CF})$, liquid water path (LWP), and aerosol indirect effect (AIE), respectively.

more, the association of a larger difference with a larger $x_{c}$ explains why the effect of $\varepsilon$ is stronger for the "POLL" case than for the "CLEAN" case as shown in Fig. 1.

For the "CLEAN" case, as daytime heating progresses, the cloud water is depleted so quickly (Fig. 1a and c) that the magnitude of $x_{c}$ jumps from $\ll 1$ to $\sim 1$. Consequently, the "CLEAN" clouds transform quickly from one stage (with efficient drizzle production) to another stage (between drizzling and non-drizzling). But for the "POLL" case, due to their continental origin and the strong large-scale subsidence (Guo et al., 2007b), these "POLL" clouds tend to precipitate less efficiently. Moreover, as shown in Fig. 2, the "CLEAN" and "POLL" cases span a wide range of $x_{c}$ from 0.1 to 40 , covering conditions of efficient $\left(x_{c} \ll 1\right)$, inefficient $\left(x_{c} \gg 1\right)$, and intermediate $\left(x_{c} \sim 1\right)$ precipitation production via the autoconversion process. Thus, these two cases include clouds with different precipitation efficiencies.

Compared to the significant differences in the instantaneous CF, LWP, and AIE, the differences in the corresponding daily averages are less noticeable. For various values of $\varepsilon$ from 0 to 300, the daily averaged CF and LWP differ by $<3 \%$, while the daily averaged AIE differs by up to $15 \%$. The larger difference in the AIE is due to the magnification of the differences in CF and LWP by the stronger insolation in the afternoon.

These differences in the instantaneous and averaged values imply that the influence of the "smoothness" of threshold representations (determined by $\varepsilon$ ) is scale-dependent: it is more significant for the instantaneous values than for the daily averages. Furthermore, the effect of $\varepsilon$ strongly depends on $x_{c}$ or the percentage of clouds in the transitional stage (with weak drizzle or between drizzling and non-drizzling); and the same is expected to hold true for global averages.

It is noteworthy that $\varepsilon$ also influences the rate function $P_{0}$ in Eq. (1) (note: $P_{0}=\kappa \frac{\left(1+3 \varepsilon^{2}\right)\left(1+4 \varepsilon^{2}\right)\left(1+5 \varepsilon^{2}\right)}{\left(1+\varepsilon^{2}\right)\left(1+2 \varepsilon^{2}\right)} N_{d}^{-1} \mathrm{LWC}^{3}$, 


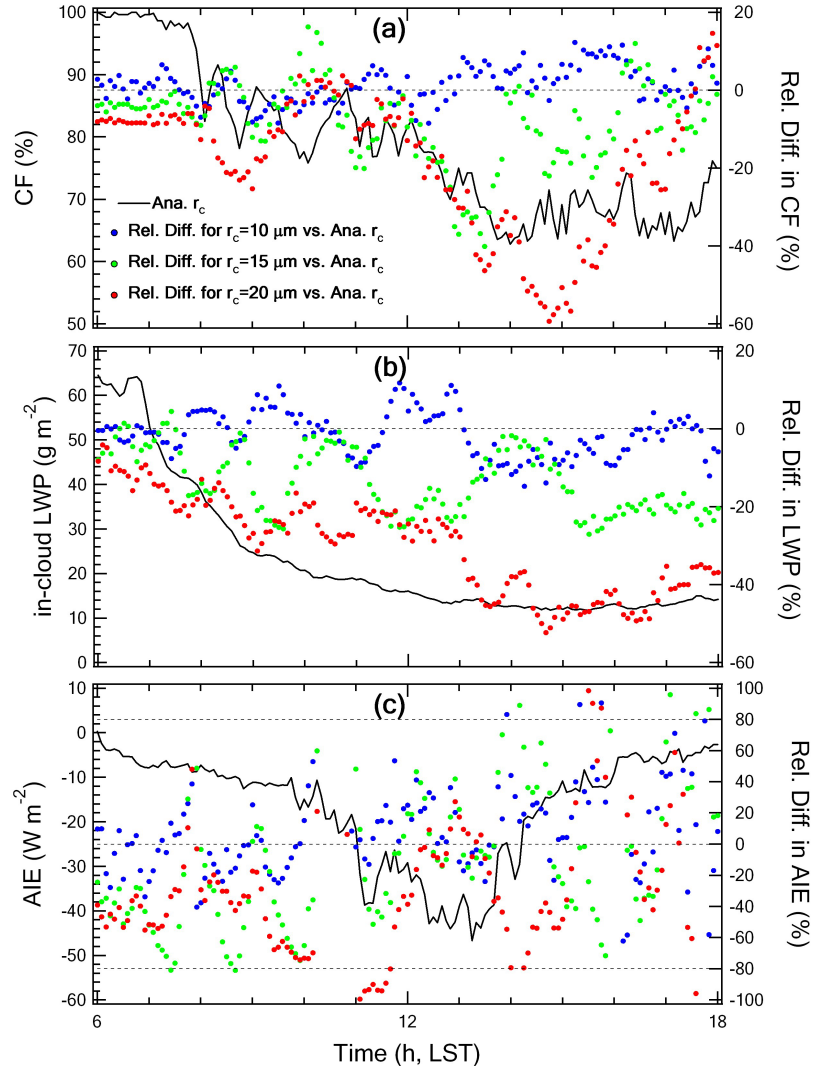

Fig. 3. Similar to Fig. 1, but for the relative differences (Rel. Diff.) between the base case with the analytical critical radius $r_{c}$ (Ana. $r_{c}$ ) and the sensitivity tests with the prescribed $r_{c}$ of $10 \mu \mathrm{m}$ (blue), $15 \mu \mathrm{m}$ (green), $20 \mu \mathrm{m}$ (red) for the "CLEAN" case.

Eq. (8) of Liu et al., 2006b). However, in order to isolate the dispersion effect on the threshold function, in this study the values of $\varepsilon$ are set to be 0.1 and 0.2 for the calculations of $P_{0}$ in the "CLEAN" and "POLL" cases, respectively (according to the observational study by Pawlowska et al., 2006).

\subsection{Sensitivity to the critical radius $\left(r_{c}\right)$}

As discussed in Sect. 2, in addition to $\varepsilon$, the theoretical threshold function also depends on the critical-to-mean mass ratio of cloud water, $x_{c}$. Since $x_{c}$ is a function of critical radius $\left(r_{c}\right)$ and $r_{c}$ is a parameter widely used in existing autoconversion parameterizations, the sensitivity to $x_{c}$ is replaced here by the sensitivity to $r_{c}$.

The critical radius $r_{c}$ has often been prescribed and/or "tuned" to achieve satisfactory agreement with observations of cloud water, albeit without a sound physical basis (Rotstayn, 1998, 2000). Recently Liu et al. (2004) derived an analytical expression for $r_{c}$ based on the kinetic potential theory (McGraw and Liu, 2003, 2004):

$r_{c}=2.8522 \times N^{1 / 6} / L^{1 / 3}$ where $r_{c}$ is in $\mu \mathrm{m}, N$ is the cloud droplet number concentration in $\mathrm{cm}^{-3}$, and $L$ is the liquid water content in $\mathrm{g} \mathrm{cm}^{-3}$. Rotstayn and Liu (2005) found that replacing the prescribed $r_{c}$ with the analytical $r_{c}$ reduced the second AIE by $\sim 27 \%$ using the CSIRO (Commonwealth Scientific and Industrial Research Organisation) GCM. A recent GCM inter-comparison study implied that the variations in the response of LWP to changes in aerosol concentrations may partly be caused by the assumed threshold values (Penner et al., 2006).

To better understand the effect of $r_{c}$ and compare it to the effect of $\varepsilon$, we have performed sensitivity tests on $r_{c}$ by applying the analytical $r_{c}$ and by prescribing $r_{c}$ to be $10,15,20$, and $25 \mu \mathrm{m}$. Figure 3 shows the results for the "CLEAN" case with $\varepsilon=0$ (i.e. the Kessler-type threshold function). The results with the analytical $r_{c}$ are shown as the base case (black), and the results with $r_{c}=10,15,20 \mu \mathrm{m}$ are shown in percentage differences relative to this base case. Evidently, a larger prescribed $r_{c}$ is associated with smaller CF and LWP. At first glance, this association seems contradictory to the hypothesis that decreased precipitation leads to larger CF and LWP (Albrecht, 1989). Deeper analysis reveals that the smaller CF and LWP are due to the stabilization of the boundary layer by precipitation formation. Smaller precipitation with increasing $r_{c}$ results in higher entrainment drying and thereby a more efficient depletion of cloud water (Ackerman et al., 2004; Bretherton et al., 2007; Guo et al., 2007b). In the afternoon, the instantaneous CF and LWP (with $r_{c}=20 \mu \mathrm{m}$ ) are reduced as much as $60 \%$ and $50 \%$, respectively. Consequently, clouds exhibit a more significant diurnal change. This is due to a positive feedback between the cloud geometric radius and entrainment. A smaller cloud radius allows cloudy air to have a larger surface area to mix with drier ambient air, and thus enhances entrainment drying (Blyth et al., 1988).

The relative difference in the instantaneous AIE is even more striking and varies by up to $\sim 80 \%$ in the daytime (Fig. 3c). The magnitude of the AIE tends to decrease with a larger $r_{c}$, corresponding to the decreased CF and LWP. The variation in the AIE tends to be larger than that in CF and in LWP.

The difference between the base case and the sensitivity test with $r_{c}=10 \mu \mathrm{m}$ is minimal, because the averaged analytical $r_{c}$ is close to $10 \mu \mathrm{m}$. A critical radius of $10 \mu \mathrm{m}$ is suggested by Pawlowska and Brenguier for the ACE-2 (2003). This good agreement provides observational support for using the analytical $r_{c}$.

Relative to the base case using the analytical $r_{c}$, the daily averaged CF, LWP, and AIE are reduced by $20 \%, 20 \%$, and $40 \%$, respectively, when the prescribed $r_{c}=20 \mu \mathrm{m}$ is used, and they are reduced by $6 \%, 6 \%$, and $20 \%$, respectively, when the prescribed $r_{c}=15 \mu \mathrm{m}$ is used as is suggested for heavily drizzling clouds (Gerber, 1996). These results highlight the high sensitivities of the simulated cloud properties and the estimated aerosol forcing to $r_{c}$ for drizzling clouds. 


\section{Discussion and concluding remarks}

The sensitivities of the cloud fraction (CF), liquid water path (LWP), and aerosol indirect effect (AIE) to different threshold representations associated with the autoconversion process were systematically examined by applying a theoretical threshold function to a 3-D cloud-resolving model.

The major results can be summarized as follows. First, the sensitivity to threshold representations is larger for the instantaneous CF, LWP, and AIE than for the corresponding daily averages. For example, for values of the droplet relative dispersion $(\varepsilon)$ of 0 and 300 , the relative differences in the instantaneous CF, LWP, and AIE are as large as $20 \%, 40 \%$, and $100 \%$, respectively; while the differences in the daily averages are generally less than $15 \%$; Second, the sensitivity depends critically on the critical-to-mean mass ratio of cloud water $\left(x_{c}\right)$, or the percentage of transitional clouds with weak drizzle or between drizzling and non-drizzling; Third, the sensitivity to critical radius $\left(r_{c}\right)$ is more significant than the sensitivity to the "smoothness" of threshold representations as embodied in $\varepsilon$ for drizzling clouds; Fourth, both CF and LWP tend to vary most significantly during the local afternoon when different values of $\varepsilon$ and $r_{c}$ are used. The magnification of the variations in CF and LWP by the stronger insolation near local noon leads to a larger variation in the AIE. Therefore, the relative differences in the AIE are larger than those in CF and LWP.

Two points are of note in passing. First, although the theoretical threshold function as given by Eq. (2) is desirable in general modelling studies (Lohmann et al., 2007), further development is needed to combine it with the rate function $P_{0}$ and the assumed sub-grid moisture distributions (e.g. symmetric triangular distribution of total water mixing ratio, Gaussian distribution of mixing ratio of suspended water (i.e. vapor and cloud water)) (personal communications with L. D. Rotstayn and A. Chen, 2007). Moreover, it might be more complex than is warranted in current GCMs since simplified representations of cloud fraction are often used. In order to explore the potential for studying the effect of different threshold representations in GCMs, we have also conducted similar sensitivity tests using an approximate alternative, the generalized Sundqvist threshold function (Liu et al., 2006b), and we have obtained similar results (not shown here). Second, this study covers all existing types of ad hoc threshold functions, but the effect of $\varepsilon$ here should not be considered to be the total effect of $\varepsilon$ on clouds and/or aerosol radiative forcing, because the current expression for $r_{c}$ (and thus $x_{c}$ ) does not account for $\varepsilon$ explicitly (Liu et al., 2004), and the effect of $\varepsilon$ on the rate function $P_{0}$ has not been applied in this study.

Acknowledgements. H. Guo and Y. Liu are supported by the Atmospheric Radiation Measurements (ARM) Program and Atmospheric Sciences Program (ASP) of the U.S. Department of Energy under Contract No. DE-AC02-98CH1-886. J. E. Penner acknowledges support by the ARM Program under grant DOE-DEFG02-97-ER62370.

Edited by: U. Lohmann

\section{References}

Ackerman, A. S., Kirkpatrick, M. P., Stevens, D. E., and Toon, O. B.: The impact of humidity above stratiform clouds on indirect aerosol climate forcing, Nature, 432, 1014-1017, 2004.

Albrecht, B. A.: Aerosols, cloud microphysics, and fractional cloudiness, Science, 243, 1227-1230, 1989.

Beheng, K. D.: A parameterization of warm cloud microphysical conversion processes, Atmos. Res., 33, 193-206, 1994.

Berry, E. X.: Modification of the warm rain process. Preprints, First National Conf. on Weather Modification, Am. Meteor. Soc., Albany, NY, 81-88, 1968.

Blyth, A. M., Cooper, W. A., and Jensen, J. B.: A study of the source of entrained air in Montana cumuli, J. Atmos. Sci., 45, 3944-3964, 1988.

Boucher, O., LeTreut, H., and Baker, M. B.: Precipitation and radiation modelling in a GCM: Introduction of cloud microphysical processes, J. Geophys. Res., 100, 16395-16414, 1995.

Bretherton, C. S., Blossey, P. N., and Uchida J.: Cloud droplet sedimentation, entrainment efficiency, and subtropical stratocumulus albedo, Geophys. Res. Lett., 34, L03813, doi:10.1029/2006GL027648, 2007.

Del Genio, A. D., Yao, M., Kovari, W., and Lo, K. K.: A prognostic cloud water parameterization for climate models, J. Climate, 9, 270-304, 1996.

Gerber, H.: Microphysics of marine stratocumulus clouds with two drizzle modes, J. Atmos. Sci., 53, 1649-1662, 1996.

Guibert, S., Snider J. R., and Brenguier, J.-L.: Aerosol activation in marine stratocumulus clouds 1 . Measurement validation for a closure study, J. Geophys. Res., 108, 8628, doi:10.1029/2002JD002678, 2003.

Guo, H., Penner, J. E., Herzog, M., and Xie, S.: Investigation of the first and second aerosol indirect effects using data from the May 2003 Intensive Operational Period at the Southern Great Plains, J. Geophys. Res., 112, D15206, doi:10.1029/2006JD007173, 2007a.

Guo, H., Penner, J. E., Herzog, M., and Pawlowska, H.: Examination of the aerosol indirect effect under contrasting environments during the ACE-2 experiment, Atmos. Chem. Phys., 7, 535-548, $2007 b$.

Herzog, M., Graf, H., Textor, C., and Oberhuber, J. M.: The effect of phase changes of water on the development of volcanic plumes, J. Volcanol. Geoth. Res., 87, 55-74, 1998.

Herzog, M., Oberhuber, J. M., and Graf, H. F.: A prognostic turbulence scheme for the non-hydrostatic plume model, J. Atmos. Sci., 60, 2783-2796, 2003.

Iacobellis, S. F. and Somerville, R. C.: Evaluating parameterizations of the autoconversion process using a singlecolumn model and Atmospheric Radiation Measurement Program measurements, J. Geophys. Res., 111, D02203, doi:10.1029/2005JD006296, 2006.

Kessler, E.: On the distribution and continuity of water substance in atmospheric circulation, Meteor. Monogr., 10, 84 pp., 1969. 
Liu, Y., Daum, P. H., and McGraw, R.: An analytical expression for predicting the critical radius in the autoconversion parameterization, Geophys. Res. Lett., 31, L06121, doi:10.1029/2003GL019117, 2004.

Liu, Y., Daum, P. H., and McGraw, R.: Size truncation effect, threshold behavior, and a new type of autoconversion parameterization, Geophys. Res. Lett., 32, L11811, doi:10.1029/2005GL022636, 2005.

Liu, Y., Daum, P. H., McGraw, R., and Miller, M.: Generalized threshold function accounting for effect of relative dispersion on threshold behavior of autoconversion process, Geophys. Res. Lett., 32, L11804, doi:10.1029/2005GL025500, 2006a.

Liu, Y., Daum, P. H., McGraw, R., and Miller, M.: Parameterization of the autoconversion process. PART II: generalization of Sundqvist-type parameterizations, J. Atmos. Sci., 63, 11031109, 2006b.

Lohmann, U., Feichter, J., Chuang, C. C., and Penner, J. E.: Prediction of the number of cloud droplets in the ECHAM GCM, J. Geophys. Res., 104, 9169-9198, 1999.

Lohmann, U., Quaas, J., Kinne, S., and Feichter, J.: Different approaches for constraining global climate models of the anthropogenic indirect aerosol effect, B. Am. Meteor. Soc., 88, 243249, 2007.

Manton, M. J. and Cotton, W. R.: Formulation of approximate equations for modeling moist deep convection on the meso-scale, Atmos. Sci. Pap., 266, Dept. of Atmos. Sci., Colo. State Univ., Fort Collins, CO, 1977.

McGraw, R. and Liu, Y.: Kinetic potential and barrier crossing: a model for warm cloud drizzle formation, Phys. Rev. Lett., 90, 1, doi:10.1103/PhysRevLett.90.018501, 2003.

McGraw, R. and Liu, Y.: Analytical formulation and parameterization of the kinetic potential theory for drizzle formation, Phys. Rev., E70, 031606-1-031606-13, 2004.

Pawlowska, H. and Brenguier, J.-L.: An Observational study of drizzle formation in stratocumulus clouds for general circulation model (GCM) parameterizations, J. Geophys. Res., 108, 8630, doi:10.1029/2002JD002679, 2003.
Pawlowska, H., Grabowski, W. W., and Brenguier, J.-L.: Observations of the width of cloud droplet spectra in stratocumulus, Geophys. Res. Lett., 33, L19810, doi:10.1029/2006GL026841, 2006.

Penner, J. E., Quaas, J., Storelvmo, T., Takemura, T., Boucher, O., Guo, H., Kirkevåg, A., Kristjánsson, J. E., and Seland, Ø.: Model intercomparison of indirect aerosol effects, Atmos. Chem. Phys., 6, 3391-3405, 2006, http://www.atmos-chem-phys.net/6/3391/2006/.

Pincus, R. and Baker, M. B.: Effect of precipitation on the albedo susceptibility of clouds in the marine boundary layer, Nature, 372, 250-252, 1994.

Rotstayn, L. D.: A physically based scheme for the treatment of stratiform clouds and precipitation in large-scale models, II, Comparison of modelled and observed climatological fields, Q. J. Roy. Meteorol. Soc., 124, 389-415, 1998.

Rotstayn, L. D.: On the "tuning" of autoconversion parameterizations in climate models, J. Geophys. Res., 105, 15 495-15 507, 2000.

Rotstayn, L. D. and Liu, Y.: A smaller global estimate of the second indirect aerosol effect, Geophys. Res. Lett., 32, L05708, doi:10.1029/2004GL021922, 2005.

Snider, J. R., Guibert, S., Brenguier, J.-L., and Putaud, J.-P.: Aerosol activation in marine stratocumulus clouds: 2. Kohler and parcel theory closure studies, J. Geophys. Res., 108, 8629, doi:10.1029/2002JD002692, 2003.

Sundqvist, H.: A parameterization scheme for non-convective condensation including prediction of cloud water content, Q. J. Roy. Meteorol. Soc., 104, 677-690, 1978.

Verver, G., Raes, F., Vogelezang, D., and Johnson, D.: The 2nd aerosol characterization experiment (ACE-2) meteorological and chemical context, Tellus, 52B, 126-140, 2000. 